\title{
Residual Stress Characterization on the Mesoscale in Additive Manufacturing
}

\author{
Kathryn A. Small ${ }^{1}$, Zach Clayburn ${ }^{2}$, Jon Almer ${ }^{3}$, Jon Tischler ${ }^{3}$, David Fullwood ${ }^{2}$, Mitra L. Taheri ${ }^{1}$
}

1. Drexel University, Materials Science and Engineering Department, Philadelphia, PA, USA.

2. Brigham Young University, Mechanical Engineering Department, Provo, UT, USA.

3. Argonne National Laboratory, X-ray Science Division, Argonne, IL, USA.

Residual stress in advanced manufacturing techniques including additive manufacturing (AM) has become an important area of research with the increasing popularity of these methods. Because of the specialized environments in which AM takes place there are factors influencing the microstructure and morphology at the grain and subgrain scale which are not present in conventional manufacturing techniques [1]. Powder bed fusion processes such as direct metal laser sintering (DMLS) and selective laser melting (SLM) introduce high thermal gradients and quick cooling times which increase heterogeneity on the mesoscale (between 0.1 and $10 \mu \mathrm{m}$ ) in polycrystalline materials including residual stress and strain on this scale $[1,2]$.

In order to characterize residual stress on the mesoscale, specialized techniques are required [2]. Neutron diffraction (ND) and X-ray diffraction (XRD) have been used extensively to characterize residual stress on the macroscale and are known to be reliable, accurate methods of analysis. On the atomistic scale, the TopSpin method used with TEM is an accurate way to characterize the residual stress of polycrystalline metals. However, the gap exists in characterizing residual stress on the meso and microscales, which is larger than individual dislocations but smaller than the scale of continuum mechanics [2]. This scale is, however, the scale wherein much of the microstructure evolution takes place including grain size and shape, boundary movement, and nucleation.

The EBSD technique has recently gained interest in the residual stress characterization community for its potential in orientation and strain analysis [3]. The HR-EBSD method arrives at area maps of residual stress (Figure 1a) by using Kikuchi patterns stored during an EBSD scan to determine changes in elastic strain and lattice rotation between a reference pattern and test patterns, and is based on the distortions to the crystal lattice caused by elastic strain variations within a crystal [3]. Similarly, Synchrotron X-ray sources are capable of providing brilliant, high-energy X-rays with high penetration depths and resulting in topography-like area maps (Figure 1b) of residual stress over time, allowing researchers to observe effects of various stimuli on microstructural evolution [4]. By lowering the energy of the synchrotron Xrays, it is possible to improve the spatial resolution to be comparable to that achieved by HR-EBSD, thereby arriving at a reliable reference for an HR-EBSD map, as the HR-EBSD technique is relatively new.

Of interest in this work is the change in residual stress of an additively manufactured part before and after post-processing such as being cut from the build plate or being stress relieved by annealing. It is known that post-processing of an as-built additively manufactured part will change the stress state of the various grain at micro- to macro- level, thereby causing distortion or detachment from support structures [5], but the effect of this residual stress change on the microstructure of a part has not yet been investigated. Using the Synchrotron X-ray source at Argonne National Laboratory, area maps of the as-built residual stress are compared to that of the same part when cut from the build plate. Comparison between surface prepared 
EBSD and low-energy Synchrotron XRD maps are compared and the effect of post-processing on the microscale residual stress distribution and microstructure of a single part is discussed.

References:

[1]. Zinovieva, O., Zinoviev, A., Ploshikhin, V. Computational Materials Science 141 (2018), p. 207.

[2]. Tamura, N. et al. Review of Scientific Instruments 73 (2002).

[3]. Britton, T.B., and Wilkinson, A.J. Ultramicroscopy 114 (2012).

[4]. Withers, P.J. Practical Residual Stress Measurement Methods. Wiley \& Sons, Inc. (2013).

[5]. Wu, A.S. et al. Metallurgical and Materials Transactions A 45 (2014).
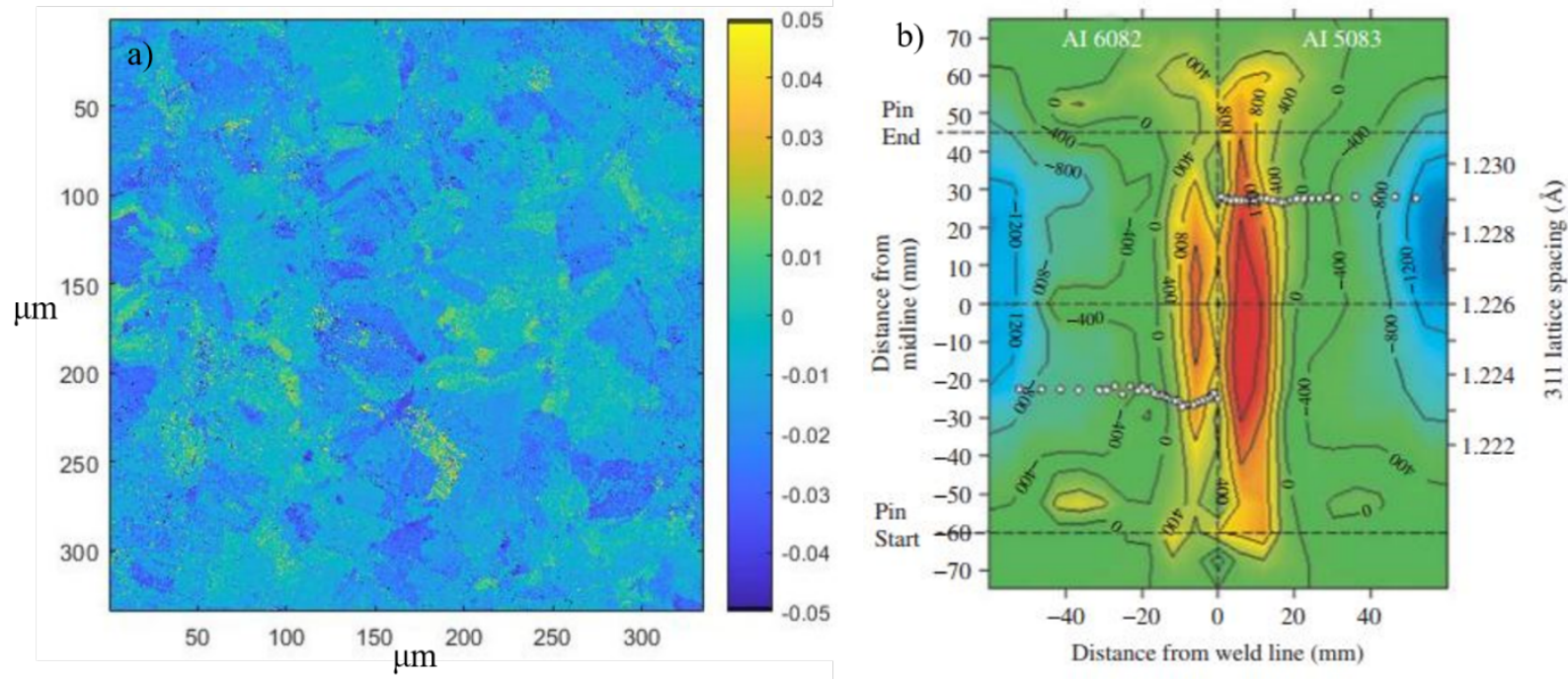

Figure 1. Example residual stress maps achieved by a) HR-EBSD method and b) Synchrotron X-rays 